\title{
Molecular junctions in the Coulomb blockade regime: Rectification and nesting
}

\author{
Bo Song, Dmitry A. Ryndyk, and Gianaurelio Cuniberti \\ Institute for Theoretical Physics, University of Regensburg, D-93040 Regensburg, Germany \\ (Received 16 November 2006; revised manuscript received 8 March 2007; published 12 July 2007)
}

\begin{abstract}
Quantum transport through single molecules is very sensitive to the strength of the molecule-electrode contact. Here, we investigate the behavior of a model molecular junction weakly coupled to external electrodes in the case where charging effects do play an important role (Coulomb blockade regime). As a minimal model, we consider a molecular junction with two spatially separated donor and acceptor sites. Depending on their mutual coupling to the electrodes, the resulting transport observables show well defined features such as rectification effects in the $I-V$ characteristics and nesting of the stability diagrams. To be able to accomplish these results, we have developed a theory which allows us to explore the charging regime via the nonequilibrium Green function formalism, and found full agreement with the master-equation results. Our theory, beyond its experimental relevance, offers a transparent framework for the systematic and modular inclusion of a richer physical phenomenology.
\end{abstract}

DOI: 10.1103/PhysRevB.76.045408

PACS number(s): 85.65.+h, 73.23.- b, 73.23.Hk, 85.30.Kk

\section{INTRODUCTION}

Single-molecule electronics ${ }^{1-3}$ has been mostly investigated in the high temperature and strong contact to the electrode regime. The opposite limit of low temperature and weakly coupled molecular junctions poses a challenge to the currently available experimental techniques. Still, the possibility to probe the spectroscopy of single-molecule junctions via a lateral gate could offer new insights to the peculiar coupling of the electrical and mechanical degrees of freedom at the nanoscale, and first experiments have appeared in the literature. ${ }^{4}$ In order to be able to establish the transport mechanisms governing such molecular junctions in the Coulomb blockade (CB) regime, a technique which could tackle on one hand single electron charging effects and, on the other hand, the inclusion of the electron-vibron coupling is of extreme importance. The nonequilibrium Green function (NEGF) formalism has been recently employed to describe transport observables on the base of a density functional theory description of the electronic structure ${ }^{3,5-11}$ and model Hamiltonian approaches. ${ }^{12-15}$ Previously, we applied the NEGF to describe the influence of the vibron dynamics onto a molecular transistor, ${ }^{16,17}$ but the NEGF method is typically substituted with master-equation approaches when coming to the case of the Coulomb blockade. ${ }^{18-20}$ Our purpose is to study the problem of a two-site donor and/or acceptor molecule in the CB regime within the NEGF as a first step to deal with the phenomenology of a rigid multilevel island. The nuclear dynamics (vibrations) always present in molecular junctions could then be modularly included in this theory. Our method developed in this paper can be calibrated on the well-studied double quantum dot problem ${ }^{21,22}$ and could be possibly integrated in the density functional theory based approaches to molecular conductance.

Here, we apply our theory to the case of a two site energetically asymmetric molecular junction. In the case of serial coupling to the electrodes, this configuration consists, de facto, in a molecular rectifier (diode) as proposed a long time ago by Aviram and Ratner $^{23}$ and recently experimentally realized. ${ }^{24}$ We show that the sequential tunneling regime, be- ing a fundamental different regime from coherent transport, is compatible to the observed rectification features. ${ }^{24}$ The serial arrangement of a double-site correlated molecule between two leads is possibly the simplest configuration. The more general case (see Fig. 1), which includes parallel pathways, shows in the sequential tunneling regime an interplay of correlated effect and interference eventually bringing to the phenomenon of a nesting of the stability diagrams due to the coexistence of different charging energies.

In this paper, we introduce a powerful ansatz for the NEGF which is related both to the equation-of-motion (EOM) method and to the Dyson equation approach. From the knowledge of the Green function (GF), we then calculate the transport observables. Our results are of particular interest in its own at a formal level. In the case of a single site junction (SSJ) with Coulomb interaction (Anderson impurity model), the linear conductance properties have been successfully studied by means of the EOM approach in the cases related to $\mathrm{CB}^{25,26}$ and the Kondo effect. ${ }^{27}$ Later, the same method was applied to some two-site models. ${ }^{12,28,29}$ Multilevel systems were started to be considered only recently. ${ }^{30,31}$ Besides, there are some difficulties in building the lesser GF in the nonequilibrium case (at finite bias voltages) by means of the EOM method. ${ }^{32-34}$ Here, we develop a self-consistent nonequilibrium method for the GF of a SSJ and of a doublesite junction (DSJ). The results of the EOM method could be calibrated with other available calculations, such as the

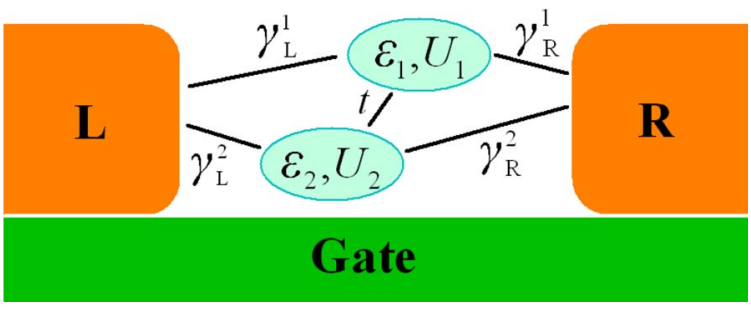

FIG. 1. (Color online) The general configuration of a doublesite junction. The levels $\epsilon_{1,2}$ with charging energies $U_{1,2}$ are connected via $t$ and coupled to the electrodes via the linewidth injection rates $\gamma_{\alpha}^{i}$. 
master-equation approach and the noncrossing approximation. This paper is organized as follows: after the derivation of the nonequilibrium results for the retarded and lesser GFs for the SSJ and DSJ (Sec. II), we do show their effects on the transport observables (Sec. III).

\section{NONEQUILIBRIUM EOM METHOD}

The goal of this paper is the determination of the transport observables for a minimal model of a molecular junction in the CB regime, namely, a double-site correlated impurity Hamiltonian coupled to extended electrode states. For clarity, we first describe our method in the more familiar problem of a single site junction, which is the well-known Anderson impurity model.

\section{A. Single site case}

The Anderson impurity model is used to describe the Coulomb interaction on a single site:

$$
H=H_{D}+\sum_{\alpha}\left(H_{\alpha}+H_{\alpha D}\right),
$$

where

$$
\begin{gathered}
H_{D}=\sum_{\sigma}\left(\epsilon_{\sigma} d_{\sigma}^{\dagger} d_{\sigma}+\frac{1}{2} U n_{\sigma} n_{\bar{\sigma}}\right), \\
H_{\alpha}=\sum_{k, \sigma} \epsilon_{k, \sigma}^{\alpha} c_{\alpha, k, \sigma}^{\dagger} c_{\alpha, k, \sigma}, \\
H_{\alpha D}=\sum_{k, \sigma}\left(V_{\alpha, k, \sigma} c_{\alpha, k, \sigma}^{\dagger} d_{\sigma}+V_{\alpha, k, \sigma}^{*} d_{\sigma}^{\dagger} c_{\alpha, k, \sigma}\right),
\end{gathered}
$$

where $d$ and $c$ are the operators for electrons on the dot and on the left $(\alpha=L)$ and the right $(\alpha=R)$ lead, $U$ is the Coulomb-interaction parameter, $\epsilon_{\sigma}$ is the $\sigma$ level of the quantum dot, while $\epsilon_{k, \sigma}^{\alpha}$ is the spin $\sigma$ level of lead $\alpha$ in $k$ space, $\sigma=\uparrow, \downarrow$. With the help of the EOM and the truncation approximation, we can get a closed set of equations for the retarded and advanced GFs $G_{\sigma, \tau}^{r / a}{ }^{26,35}$

$$
\begin{aligned}
& \left(\omega-\epsilon_{\sigma}-\Sigma_{\sigma}^{r / a}\right) G_{\sigma, \tau}^{r / a}=\delta_{\sigma, \tau}+U G_{\sigma, \tau}^{(2) r / a}, \\
& \left(\omega-\epsilon_{\sigma}-U-\Sigma_{\sigma}^{r / a}\right) G_{\sigma, \tau}^{(2) r / a}=\left\langle n_{\bar{\sigma}}\right\rangle \delta_{\sigma, \tau},
\end{aligned}
$$

where $G_{\sigma, \tau}^{r / a}=\left\langle\left\langle d_{\sigma} \mid d_{\tau}^{\dagger}\right\rangle\right\rangle^{r / a}, G_{\sigma, \tau}^{(2) r / a}=\left\langle\left\langle n_{\bar{\sigma}} d_{\sigma} \mid d_{\tau}^{\dagger}\right\rangle\right\rangle^{\gamma / a}$, and

$$
\Sigma_{\sigma}^{r / a}(\omega)=\Sigma_{L, \sigma}^{r / a}+\Sigma_{R, \sigma}^{r / a}=\sum_{\alpha, k} \frac{\left|V_{\alpha, k, \sigma}\right|^{2}}{\omega-\epsilon_{k, \sigma}^{\alpha} \pm \mathrm{i} 0^{+}}
$$

are the electron self-energies.

\section{B. Nonequilibrium second-order truncation}

\section{Mapping on retarded Green functions}

There are two typical ways to calculate GFs. The first is by means of the Dyson equation and Feynman diagrams, the second is by means of the EOM. ${ }^{36}$ For retarded GFs, from the EOM method, and with the help of Eqs. (5a) and (5b), we can get

$$
G^{r}=G_{0}^{r}+G_{0}^{r} U G^{(2) r}=G_{0}^{r}+G_{0}^{r} \Sigma^{\mathrm{EOM}} G^{(1) r},
$$

where $G^{r}$ is the single-particle GF matrix

$$
G^{r}=\left(\begin{array}{cc}
G_{\uparrow, \uparrow}^{r} & G_{\uparrow, \downarrow}^{r} \\
G_{\downarrow, \uparrow}^{r} & G_{\downarrow, \downarrow}^{r}
\end{array}\right),
$$

and $G_{\sigma, \tau}^{(1) r}=G_{\sigma, \tau}^{(2) r} /\left\langle n_{\bar{\sigma}}\right\rangle . G_{0}^{r}$ describes the single-particle spectrum without Coulomb interaction, but including the effects from the electrodes. $\Sigma_{\sigma, \tau}^{\mathrm{EOM}}=U\left\langle n_{\bar{\sigma}}\right\rangle$ is the Hartree-like selfenergy of our model. Since there is only Coulomb interaction on the site with the levels $\epsilon_{\sigma}$, the Fock-like self-energy is vanishing.

Alternatively, by means of the Dyson equation and the second-order truncation approximation, taking Hartree-like self-energies $\Sigma_{\sigma, \tau}^{H}=U\left\langle n_{\bar{\sigma}}\right\rangle\left(=\Sigma_{\sigma, \tau}^{\mathrm{EOM}}\right)$, we can also get the retarded GFs as follows: ${ }^{36}$

$$
G^{r}=G_{0}^{r}+G_{0}^{r} \Sigma^{H} G_{1}^{r},
$$

where $G_{1}^{r}=G_{0}^{r}+G_{0}^{r} \Sigma^{H} G_{0}^{r}$ is the first-order truncation GF. Within the level of the second-order truncation approximation, we see that there is a map between the EOM results and the Dyson results:

$$
\begin{aligned}
& G^{r}=G_{0}^{r}+G_{0}^{r} \Sigma^{H} G^{(1) r} \quad(\mathrm{EOM}), \\
& \uparrow \quad \uparrow \\
& G^{r}=G_{0}^{r}+G_{0}^{r} \Sigma^{H} G_{1}^{r} \quad \text { (Dyson). }
\end{aligned}
$$

Equation (10) prompts a way to include further manyparticle effects into the Dyson equation, Eq. (10b), by replacing the Dyson-first-order retarded Green function $G_{1}^{r}$ with the EOM $G^{(1) r}$. Then, one already obtains the correct results to describe $\mathrm{CB}$ while keeping the Hartree-like self-energy.

\section{Mapping on contour and lesser Green functions}

Introducing now the contour GF $\hat{G}$, we can get the Dyson equation as follows: ${ }^{35,37-39}$

$$
\check{G}=\check{G}_{0}+\check{G}_{0} \check{\check{\Sigma}} \check{G},
$$

where $\Sigma$ is the self-energy matrix. ${ }^{35}$ According to the approximation for the retarded GF in Eq. (9), we take the second-order truncation on Eq. (11), and then get

$$
\check{G}=\check{G}_{0}+\check{G}_{0} \check{\check{\Sigma}}^{H} \check{G}_{1},
$$

where $\check{G}_{1}=\check{G}_{0}+\check{G}_{0} \check{\Sigma}^{H} \check{G}_{0}$ is the first-order contour GF, and $\check{G}_{0}$ has already included the lead broadening effects.

Similar to the mapping in Eq. (10), we perform an ansatz consisting in substituting the Dyson-first-order $G_{1}^{r / a /<}$ with the EOM one $G^{(1) r / a /<}$ to consider more many-particle correlations, while the EOM self-energy is used for the Dyson equation for consistency: 


$$
\begin{array}{lll}
\check{G}=\check{G}_{0}+\check{G}_{0} \check{\Sigma}^{\check{\Sigma}^{H}} & \check{G}_{1} & \text { (Dyson), } \\
\downarrow & \uparrow & \\
\check{G} & \check{G}^{(1)} & (\mathrm{EOM}) .
\end{array}
$$

Then, using the Langreth theorem, ${ }^{35}$ we get the lesser GF,

$$
\begin{aligned}
G^{<} & =G_{0}^{<}+G_{0}^{r} \Sigma^{H, r} G^{(1)<}+G_{0}^{<} \Sigma^{H, a} G^{(1) a} \\
& =G_{0}^{<}+G_{0}^{r} U G^{(2)<}+G_{0}^{<} U G^{(2) a},
\end{aligned}
$$

where $G_{0}^{r / a l<}$ are GFs for $U=0$, but including the lead broadening effects, i.e.,

$$
\begin{gathered}
G_{0}^{<}=g_{0}^{<}+g_{0}^{r} \Sigma^{<} G_{0}^{a}+g_{0}^{<} \Sigma^{a} G_{0}^{a}+g_{0}^{r} \Sigma^{r} G_{0}^{<}, \\
G_{0}^{r / a}=g_{0}^{r / a}+g_{0}^{r / a} \Sigma^{r / a} G_{0}^{r / a},
\end{gathered}
$$

with $g_{0}^{r / a l<}$ the free electron GF, and

$$
\Sigma^{r / a l<}=\left(\begin{array}{cc}
\sum_{\uparrow}^{r / a l<} & 0 \\
0 & \sum_{!}^{r / a /<}
\end{array}\right),
$$

$\Sigma_{\sigma}^{<}=i \Sigma_{\alpha} \Gamma_{\alpha} f_{\alpha}(\omega)$, and $\Gamma_{\alpha}=i\left(\Sigma_{\alpha}^{r}-\Sigma_{\alpha}^{a}\right), f_{\alpha}(\omega)=f\left(\omega-\mu_{\alpha}\right), f$ is the equilibrium Fermi function, and $\mu_{\alpha}$ is the electrochemical potential in lead $\alpha ; \Sigma_{\alpha}^{r / a}$ are the retarded and/or advanced electron self-energies from Eq. (6) and $G_{\sigma, \tau}^{(1) r / a /<}$ $=G_{\sigma, \tau}^{(2) r / a /<} /\left\langle n_{\bar{\sigma}}\right\rangle$. Performing the same ansatz on the doubleparticle GF, from Eq. (5b), we can get

$$
G^{(2)<}=G^{(2) r} \Sigma^{(2)<} G^{(2) a},
$$

with $\Sigma_{\sigma}^{(2)<}=\Sigma_{\sigma}^{<} /\left\langle n_{\bar{\sigma}}\right\rangle$.

The lesser GFs in Eq. (14) can also be obtained directly from the general formula ${ }^{35}$

$$
G^{<}(\omega)=G_{0}^{<}+G_{0}^{r} \Sigma^{r} G^{<}+G_{0}^{r} \Sigma^{<} G^{a}+G_{0}^{<} \Sigma^{a} G^{a},
$$

with the help of the ansatz in Eq. (13). It should be noted that Eq. (14) is very different from the lesser GF formula

$$
G^{<}=G^{r} \Sigma^{<} G^{a},
$$

with the self-energy $\Sigma<$ containing only contributions from the electrodes. Equation (20) is widely used for both first-principles ${ }^{5,10,40}$ and model Hamiltonian calculations. ${ }^{12}$

The numerical calculation results of conductance dependence on the bias and gate voltages by the two different

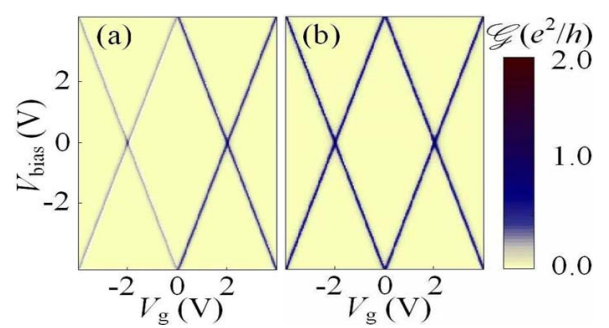

FIG. 2. (Color online) The stability diagram of a SSJ with $\epsilon_{\sigma}$ $=2.0 \mathrm{eV}, U=4.0 \mathrm{eV}, \Gamma_{L}=\Gamma_{R}=0.05 \mathrm{eV}$. (a) The incorrect result obtained by means of the widely used formula in Eq. (20) for the lesser GF is not symmetric for levels $\epsilon_{\sigma}$ and $\epsilon_{\sigma}+U$. (b) Results obtained by means of our ansatz in Eq. (14) shows correctly symmetric for levels $\epsilon_{\sigma}$ and $\epsilon_{\sigma}+U$.

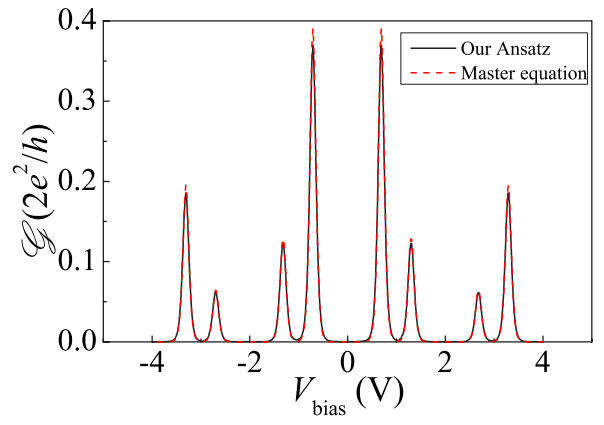

FIG. 3. (Color online) The comparison of the master-equation method and our ansatz for the differential conductance of the two level model with $\epsilon_{\uparrow}=-0.35 \mathrm{eV}, \epsilon_{\downarrow}=-0.65 \mathrm{eV}, U=1.0 \mathrm{eV}, V_{g}$ $=1.0 \mathrm{~V}$, and $\Gamma_{L}=\Gamma_{R}=0.05 \mathrm{eV}$.

NEGFs [Eqs. (14) and (20)] are shown in Fig. 2. As we can see in the left panel, the adoption of Eq. (20) results in an incorrectly symmetry breaking in the gate potential. This wrong behavior is corrected in the right panel where Eq. (14) has been used. Note that the expressions for the retarded and lesser functions, described above, can be entirely obtained by means of the EOM method formulated on the Keldysh contour (see the Appendix).

\section{Comparison with the master-equation result}

In the single site model with two (spin-up and spin-down) levels, it is possible to make the direct comparison between our ansatz and the master-equation methods. For the latter, we used the well-known master-equation technique for quantum dots. ${ }^{41,42}$

In Fig. 3, the typical curves of the differential conductance as a function of the bias voltage at fixed gate voltage obtained by the two methods are shown together: there is basically no difference in the results obtained by these two methods. In Fig. 4, the contour plot of the differential conductance obtained by our ansatz is shown. We do not present here the contour plot obtained by the master-equation method because it looks exactly the same.

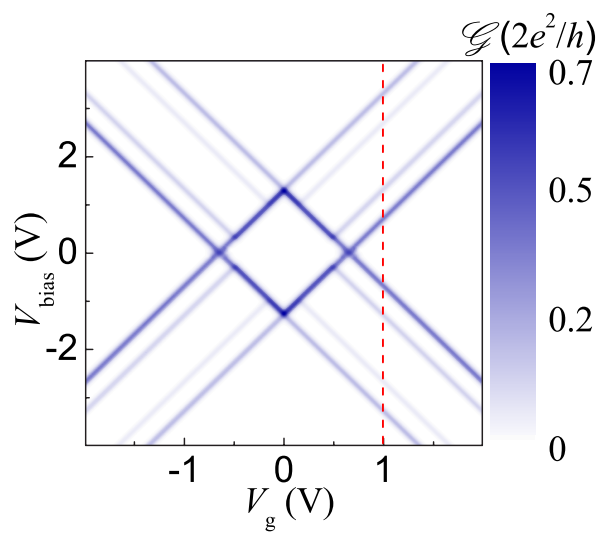

FIG. 4. (Color online) The stability diagram (the contour plot of the differential conductance) calculated by our ansatz for the two level model with parameters as in Fig. 3. The latter is indicated with a dash line at $V_{g}=1.0 \mathrm{~V}$. 
It is quite clear from the presented figures that our ansatz and the master-equation method give essentially the same results in the limit of weak coupling to the leads. The systematic investigation of the deviations between the two methods at stronger tunneling will be presented in a separate publication.

It is important that our ansatz can be applied straightforwardly to multilevel systems in the case when the exact eigenstates of an isolated system are unknown and the usage of the master-equation method is not easy. In this paper, we consider the simplest example of such a system, namely, a double-site case.

\section{Double-site case}

We now return to the investigation of the DSJ system (Fig. 1) with Coulomb interaction on each site. The Hamiltonian is expressed as follows:

$$
H=H_{D}+H_{t}+\sum_{\alpha}\left(H_{\alpha}+H_{\alpha D}\right)
$$

where

$$
\begin{gathered}
H_{D}=\sum_{i, \sigma}\left(\epsilon_{i, \sigma} d_{i, \sigma}^{\dagger} d_{i, \sigma}+\frac{U_{i}}{2} n_{i, \sigma} n_{i, \bar{\sigma}}\right), \\
H_{t}=\sum_{i \neq j, \sigma} \frac{t}{2}\left(d_{i, \sigma}^{\dagger} d_{j, \sigma}+d_{j, \sigma}^{\dagger} d_{i, \sigma}\right), \\
H_{\alpha, \sigma}=\sum_{k, \sigma} \epsilon_{k, \sigma}^{(\alpha)} c_{\alpha, k, \sigma}^{\dagger} c_{\alpha, k, \sigma}, \\
H_{\alpha D, \sigma}=\sum_{k, \sigma}\left(V_{\alpha, k, \sigma} c_{\alpha, k, \sigma}^{\dagger} d_{i, \sigma}+V_{\alpha, k, \sigma}^{*} d_{i, \sigma}^{\dagger} c_{\alpha, k, \sigma}\right),
\end{gathered}
$$

with $i, j=1,2$ indicate the site, $t$ is the constant for electron hopping between different sites.

With the help of the EOM, and by means of the truncation approximation on the double-particle GFs, we obtain the closed form for the retarded GFs as follows:

$$
\begin{gathered}
\left(\omega-\epsilon_{i, \sigma}-\sum_{i, \sigma}^{r}\right) G_{i, \sigma ; j, \tau}^{(U, t) r}=\delta_{i, j} \delta_{\sigma, \tau}+U_{i} G_{i, \sigma ; j, \tau}^{(2)(U, t) r}+t G_{i, \sigma ; j, \tau}^{(U, t) r} \\
\left(\omega-\epsilon_{i, \sigma}-U_{i}-\sum_{i, \sigma}^{r}\right) G_{i, \sigma ; j, \tau}^{(2)(U, t) r}=\left\langle n_{i, \bar{\sigma}}\right\rangle \delta_{i, j} \delta_{\sigma, \tau}+t n_{i, \bar{\sigma}} G_{i, \sigma ; j, \tau}^{(U, t) r},
\end{gathered}
$$

where the DSJ retarded GFs are defined as

$$
\begin{gathered}
G_{i, j ; \sigma, \tau}^{(U, t) r}=\left\langle\left\langle d_{i, \sigma} \mid d_{j, \tau}^{\dagger}\right\rangle\right\rangle^{r}, \\
G_{i, j ; \sigma, \tau}^{(2)(U, t) r}=\left\langle\left\langle n_{i, \bar{\sigma}} d_{i, \sigma} \mid d_{j, \tau}^{\dagger}\right\rangle\right\rangle^{r} .
\end{gathered}
$$

Here, $\bar{i}$ means "not $i$," and $\Sigma_{i, \sigma}^{r}$ are the electron self-energy from leads.

From Eqs. (26a) and (26b) and performing the same ansatz as in the case of SSJ, we can obtain the DSJ lesser GFs with Coulomb-interaction effects as follows;

$$
\begin{aligned}
G^{(U, t)<}(\omega)= & {\left[1+G^{(U, t) r} \Sigma_{t}^{r}\right] G^{(U)<} \cdot\left[1+\Sigma_{t}^{a} G^{(U, t) a}\right] } \\
& +G^{(U, t) r} \Sigma_{t}^{<} G^{(U, t) a}
\end{aligned}
$$

with

$$
\Sigma_{t}^{r}=\Sigma_{t}^{a}=\left(\begin{array}{cccc}
0 & t & 0 & 0 \\
t & 0 & 0 & 0 \\
0 & 0 & 0 & t \\
0 & 0 & t & 0
\end{array}\right)
$$

and $\Sigma_{t}^{<}=0 . G^{(U)<}$ is the DSJ lesser GF with the same form as Eq. (14), but taking

$$
U=\left(\begin{array}{cccc}
U_{1} & 0 & 0 & 0 \\
0 & U_{2} & 0 & 0 \\
0 & 0 & U_{1} & 0 \\
0 & 0 & 0 & U_{2}
\end{array}\right), \quad \Gamma_{\alpha}=\left(\begin{array}{cccc}
\gamma_{\alpha}^{1} & 0 & 0 & 0 \\
0 & \gamma_{\alpha}^{2} & 0 & 0 \\
0 & 0 & \gamma_{\alpha}^{1} & 0 \\
0 & 0 & 0 & \gamma_{\alpha}^{2}
\end{array}\right),
$$

where $\gamma_{\alpha}^{j}$ indicates the linewidth function of lead $\alpha$ to site $i$, and $U_{i}$ is the charging energy at site $i . G^{r / a}$ and $G^{(2) r / a}$ are the $\mathrm{GF}$ matrices from Eqs. (26a) and (26b). Here, in order to distinguish different GFs, we introduce the subscript $(U, t)$ for the one with both Coulomb interaction $U$ and intersite hopping $t$, while $(U)$ for the one only with Coulomb interaction. For our models, the lesser GFs in Eqs. (14), (18), and (29), which are obtained with the help of our ansatz, can also be obtained by the EOM NEGF formula in Eq. (A4) or in Ref. 32 within the same truncation approximation.

\section{TRANSPORT OBSERVABLES FOR THE DOUBLE-SITE JUNCTION}

The current can be generally written as ${ }^{43}$

$$
\begin{aligned}
J= & \frac{\mathrm{i} e}{2 \hbar} \int \frac{d \omega}{2 \pi} \operatorname{Tr}\left\{\left(\Gamma_{L}-\Gamma_{R}\right) G^{(U, t)<}+\left[f_{L}(\omega) \Gamma_{L}-f_{R}(\omega) \Gamma_{R}\right]\right. \\
& \left.\times\left[G^{(U, t) r}-G^{(U, t) a}\right]\right\},
\end{aligned}
$$

where the lesser GF is given by Eq. (29). The differential conductance is defined as

$$
\mathcal{G}=\frac{\partial J}{\partial V_{\text {bias }}},
$$

where the bias voltage is defined as $V_{\text {bias }}=\left(\mu_{R}-\mu_{L}\right) / e$.

\section{A. Serial configuration}

By taking $\gamma_{L}^{2}=\gamma_{R}^{1}=0$, we obtain a serial DSJ, which could describe the kind of molecular quantum junctions like the ones studied in Ref. 24. First, at small bias voltages, the conductance with the two gate voltages $V_{\mathrm{g}_{1}}$ and $V_{\mathrm{g}_{2}}$ was calculated, and the relative stability diagram was obtained, as shown in Fig. 5. Because of the double degeneracy (spin-up and spin-down) considered for each site and electrons hopping between the dots, there are eight resonance-tunneling regions. This result is consistent with the master-equation approach. $^{21}$ 


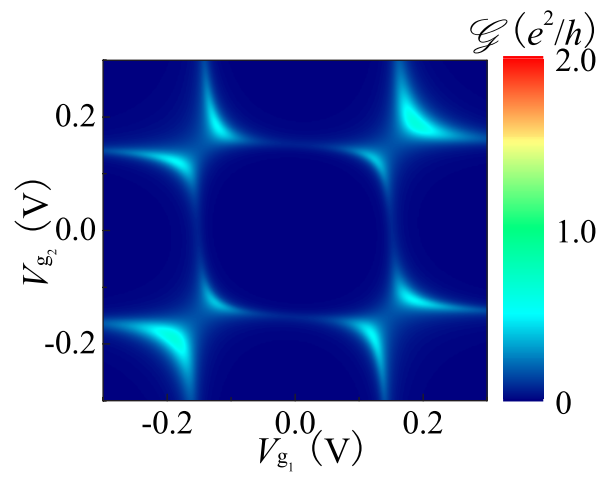

FIG. 5. (Color online) The stability diagram of a serial DSJ with $\epsilon_{1, \sigma}=\epsilon_{2, \sigma}=-0.15 \mathrm{eV}, \quad U_{1}=U_{2}=0.3 \mathrm{eV}, \quad t=0.05 \mathrm{eV}, \quad \gamma_{L}^{1}=\gamma_{R}^{2}$ $=0.02 \mathrm{eV}, \gamma_{L}^{2}=\gamma_{R}^{1}=0$, and $V_{\text {bias }}=0.005 \mathrm{~V}$. The maxima of conductance are observed when the levels of the first site $\left(\epsilon_{1, \sigma}\right.$ or $\left.\epsilon_{1, \sigma}+U\right)$ are overlapped with the levels of the second site $\left(\epsilon_{2, \sigma}\right.$ or $\left.\epsilon_{2, \sigma}+U\right)$, and with the Fermi energy in the leads. The splitting of the four maxima is due to the hopping between the dots.

Further, we studied the nonequilibrium current for large bias voltages (Fig. 6). Because $\epsilon_{1, \sigma}$ and $\epsilon_{2, \sigma}$ are taken as asymmetric, for the case without Coulomb interaction, the $I-V$ curve is asymmetric for $\pm V_{\text {bias }}$, and there are one step and one maximum for the current. The step contributes to one peak for the conductance. When we introduce the Coulomb interaction to the system, the one conductance peak is split into several: two peaks, one pseudo peak, and one dip, while the current maximum comes to be double split (see Fig. 6). The origin of this is in the effective splitting of the degenerate level when one of the spin states is occupied and the other is empty. When both spin states are occupied, the degeneracy is restored.

This process can be illustrated by the help of Fig. 7. At zero bias voltage, $\epsilon_{2, \sigma}$ is occupied and $\epsilon_{1, \sigma}$ is empty. Then, we start to increase the bias voltage. (a) The level $\epsilon_{2, \sigma}+U$ is first opened for transport. It will contribute the first peak for conductance. (b) Further, the levels $\epsilon_{2, \sigma}$ and $\epsilon_{1, \sigma}$ come into the transport window between the left and the right Fermi levels, resulting in the second peak. (c) When the level $\epsilon_{1, \sigma}$

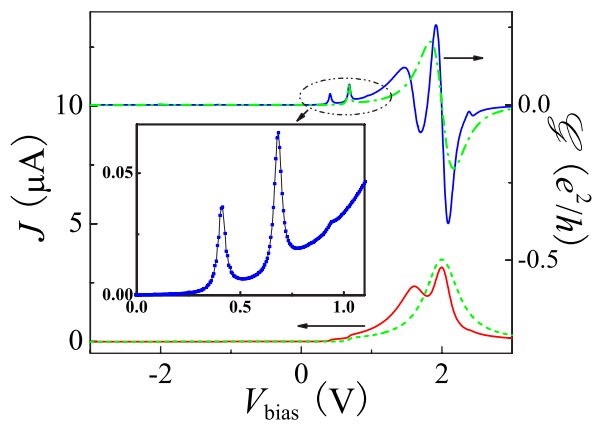

FIG. 6. (Color online) Current and conductance vs bias voltage of a DSJ far from equilibrium with parameters $\epsilon_{1, \sigma}=0.5 \mathrm{eV}$, $\epsilon_{2, \sigma}=-0.5 \mathrm{eV}, U_{1}=U_{2}=U=0.2 \mathrm{eV}, t=0.07 \mathrm{eV}, \gamma_{L}^{1}=\gamma_{R}^{2}=0.03 \mathrm{eV}$, $V_{\mathrm{g}_{2}}=-V_{\mathrm{g}_{1}}=V_{\text {bias }} / 4$, and $V_{R}=-V_{L}=V_{\text {bias }} / 2$. The red curve represents the current, while the blue the conductance. The inset is the blowup for the conductance peak split. The dash and dot-dash curves are for current and conductance with $U=0$, respectively.

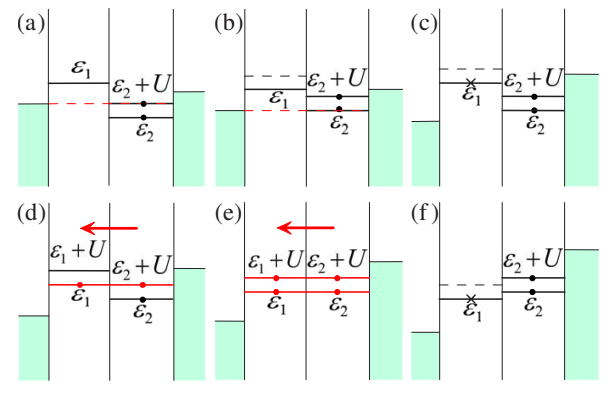

FIG. 7. (Color online) The processes involved in the transport characteristics in Fig. 6. $\epsilon_{1} \equiv \epsilon_{1, \sigma}, \epsilon_{2} \equiv \epsilon_{2, \sigma}$. The red line indicates electron resonant tunneling. (a) The first conductance peak. (b) The second conductance peak. (c) The pseudopeak of conductance. (d) The first current maximum, and the red line indicates resonant tunneling of electrons. (e) The second current maximum for electron resonant tunneling. (f) The dip of conductance.

$+U$ comes into play, only a pseudopeak appears. This is because there is only a little possibility for electrons to occupy the level $\epsilon_{1, \sigma}$ under positive bias voltage. (d) Levels $\epsilon_{2, \sigma}+U$ and $\epsilon_{1, \sigma}$ meet, which results in electron resonant tunneling and leads to the first maximum of the current. Then, a new level $\epsilon_{1, \sigma}+U$ appears over the occupied $\epsilon_{1, \sigma}$ due to the Coulomb interaction. (e) The meeting of $\epsilon_{2, \sigma}$ and $\epsilon_{1, \sigma}$ results in electron resonant tunneling. It means that $\epsilon_{1, \sigma}$ will be occupied, which leads to the appearance of a new level $\epsilon_{1, \sigma}$ $+U$. Then, $\epsilon_{2, \sigma}+U$ meets $\epsilon_{1, \sigma}+U$ and another resonant tunneling channel is opened for electrons. The two channels result in the second current maximum. (f) Finally, the level $\epsilon_{1, \sigma}+U$ disappears if the level $\epsilon_{1, \sigma}$ is empty. This means that a dip appears in the conductance. It should be noted that the characteristics of serial DSJ in Fig. 6 have showed some reasonable similarities to experiments of a single-molecule diode. $^{24}$

\section{B. Parallel configuration}

If, on the other hand, the two sites are symmetrically connected to the electrodes, possibly with a small interdot hopping, but with charging energies, $U_{1}$ and $U_{2}$ fixed to different scales for transport. The resulting stability diagram contains both interference effects for parallel pathways and an overlap of $U_{1}$ and $U_{2}$ stability diagrams, which we refer to a nesting characteristic (see Fig. 8).

The physics of the weak lines in the figure can be understood by the help of charging effects. For simplicity, here, we would ignore the site index $i$. In the region of large positive gate voltage at zero bias voltage, $\epsilon_{\uparrow}$ and $\epsilon_{\downarrow}$ are all empty, which means that the two levels are degenerate. Therefore, adding a bias voltage, first, there will be two channels $\left(\epsilon_{\uparrow}\right.$ and $\epsilon_{\downarrow}$ ) opened for current (thick lines). After then, one level $\epsilon_{\sigma}$ (spin-up or spin-down) is occupied, while the other obtains a shift for Coulomb interaction: $\epsilon_{\bar{\sigma}} \rightarrow \epsilon_{\bar{\sigma}}+U$. Therefore, when the bias voltage is further increased to make the Fermiwindow boundary meeting level $\epsilon_{\bar{\sigma}}+U$, only one channel is opened for the current, which results in the weak lines in Fig. 8 , which is the characteristic of CB. The similar case appears in the region of large negative gate voltages. 


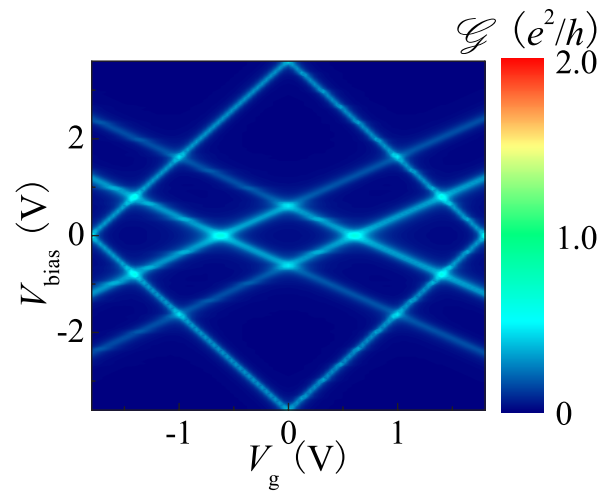

FIG. 8. (Color online) Nested stability diagram of a parallel DSJ with parameters $\epsilon_{1, \sigma}=-1.8 \mathrm{eV}, \epsilon_{2, \sigma}=-0.3 \mathrm{eV}, U_{1}=3.6 \mathrm{eV}, U_{2}$ $=0.6 \mathrm{eV}, \quad t=0.001 \mathrm{eV}, \quad \gamma_{L}^{1}=\gamma_{R}^{1}=0.04 \mathrm{eV}, \quad \gamma_{L}^{2}=\gamma_{R}^{2}=0.05 \mathrm{eV}, \quad V_{\mathrm{g}_{2}}$ $=V_{\mathrm{g}_{1}} / 2=V_{\mathrm{g}} / 2$, and $V_{R}=-V_{L}=V_{\text {bias }} / 2$. See discussion in the text.

\section{CONCLUSIONS}

In this paper, we introduced here a powerful ansatz for the lesser Green function, which is consistent with both the Dyson-equation approach and the equation-of-motion approach. By using this ansatz together with the standard equation-of-motion technique for the retarded and advanced Green functions, we obtained the NEGF for both the singleand the double-site junctions in the Coulomb blockade regime at finite voltages and calculated the transport observables. The method can be applied to describe selfconsistently transport through single molecules with strong Coulomb interaction and arbitrary coupling to the leads.

To test our method, we analyzed here the CB stability diagrams for a SSJ and a DSJ. Our results are all consistent with the results of experiments and the master-equation approach. We showed that the improved lesser Green function gives better results for weak molecule-to-contact couplings, where a comparison with the master-equation approach is possible.

For the serial configuration of a DSJ, such as a donor and/or acceptor rectifier, the $I-V$ curves maintain a diodelike behavior, as it can be already inferred by coherent transport calculations. ${ }^{44}$ Besides, we predict that as a result of charging effects, one conductance peak will be split into three peaks and one dip, and one current maximum into two. For a DSJ parallel configuration, due to different charging energies on the two dot sites, the stability diagrams show peculiar nesting characteristics. In both cases, we present the results of numerical calculations as well as the simple qualitative picture of physical processes.

We believe that the results presented here, beyond their experimental relevance, might be the transparent base for a systematic and modular inclusion of a richer physical phenomenology. Work is currently in progress to include the electron-vibron interactions to this theory.

\section{ACKNOWLEDGMENTS}

We acknowledge fruitful discussions with Rafael Gutierrez and Florian Pump. This work was funded by the Volkswagen Foundation under Grant No. I/78 340, by the Deutsche Forschungsgemeinschaft within the Priority Program SPP 1243, and the trilateral project CU 44/3-2. Support from the Vielberth Foundation is also gratefully acknowledged.

\section{APPENDIX: DERIVATION OF THE LESSER GREEN FUNCTION}

From the view of perturbation theory, our Hamiltonian can be generally written as $H=H_{0}+H_{1}$, where $H_{1}$ is the perturbation term to the solved $H_{0}$. The contour-ordered GF is defined by means of the Schwinger-Keldysh time contour

$$
\left\langle\left\langle A\left(\tau_{1}\right) ; B\left(\tau_{2}\right)\right\rangle\right\rangle^{C}=-i\left\langle T_{C}\left[A\left(\tau_{1}\right) B\left(\tau_{2}\right)\right]\right\rangle,
$$

where $A\left(\tau_{1}\right)$ and $B\left(\tau_{2}\right)$ are Heisenberg operators, defined along the contour $C$. Taking the time derivative, we obtain the EOM as

$$
\begin{aligned}
i \frac{\partial}{\partial \tau_{1}}\left\langle\left\langle A\left(\tau_{1}\right) ; B\left(\tau_{2}\right)\right\rangle\right\rangle^{C}= & \delta^{C}\left(\tau_{1}-\tau_{2}\right)\left\langle\left[A\left(\tau_{1}\right), B\left(\tau_{2}\right)\right]_{ \pm}\right\rangle \\
& +\left\langle\left\langle\left[A\left(\tau_{1}\right), H\right] ; B\left(\tau_{2}\right)\right\rangle\right\rangle^{C}
\end{aligned}
$$

Using the free particle solution $g^{\mathrm{C}}\left(\tau_{1}-\tau_{2}\right)$, we can rewrite the time-dependent solution as

$$
\begin{aligned}
\left\langle\left\langle A\left(\tau_{1}\right) ; B\left(\tau_{2}\right)\right\rangle\right\rangle^{C}= & g^{C}\left(\tau_{1}-\tau_{2}\right)\left\langle\left[A\left(\tau_{1}\right), B\left(\tau_{2}\right)\right]_{ \pm}\right\rangle \\
& +\int g^{C}\left(\tau_{1}-\tau^{\prime}\right)\left\langle\left\langle\left[A\left(\tau^{\prime}\right), H_{1}\right] ; B\left(\tau_{2}\right)\right\rangle\right\rangle^{C} d \tau^{\prime} .
\end{aligned}
$$

Now, applying the Langreth theorem and transforming in the spectral space, we get

$$
\begin{aligned}
\langle\langle A \mid B\rangle\rangle_{\omega}^{<}= & g^{<}(\omega)\left\langle[A, B]_{ \pm}\right\rangle+g^{r}(\omega)\left\langle\left\langle\left[A, H_{1}\right], B\right\rangle\right\rangle_{\omega}^{<} \\
& +g^{<}(\omega)\left\langle\left\langle\left[A, H_{1}\right], B\right\rangle\right\rangle_{\omega}^{a} .
\end{aligned}
$$

${ }^{1}$ C. Joachim, J. K. Gimzewski, and A. Aviram, Nature (London) 408, 541 (2000).

${ }^{2}$ A. Nitzan and M. A. Ratner, Science 300, 1384 (2003).

${ }^{3}$ Lecture Notes in Physics Vol. 68, edited by G. Cuniberti, G. Fagas, and K. Richter (Springer, Berlin, 2005).

${ }^{4}$ E. A. Osorio et al., Adv. Mater. 19, 281 (2007); J. Park et al., Nature (London) 417, 722 (2002).
${ }^{5}$ M. Brandbyge, J.-L. Mozos, P. Ordejon, J. Taylor, and K. Stokbro, Phys. Rev. B 65, 165401 (2002).

${ }^{6}$ J. Taylor, M. Brandbyge, and K. Stokbro, Phys. Rev. B 68, 121101(R) (2003).

${ }^{7}$ T. Frauenheim, G. Seifert, M. Elstner, Z. Hajnal, G. Jungnickel, D. Porezag, S. Suhai, and R. Scholz, Phys. Status Solidi B 217, $41(2000)$ 
${ }^{8}$ A. D. Carlo, M. Gheorghe, P. Lugli, M. Sternberg, G. Seifert, and T. Frauenheim, Physica B 314, 86 (2002).

${ }^{9}$ T. Frauenheim, G. Seifert, M. Elstner, T. Niehaus, C. K. M. Amkreutz, M. Sternberg, Z. Hajnal, A. D. Carlo, and S. Suhai, J. Phys.: Condens. Matter 14, 3015 (2002).

${ }^{10}$ A. R. Rocha, V. M. Garcia-Suarez, S. Bailey, C. Lambert, J. Ferrer, and S. Sanvito, Phys. Rev. B 73, 085414 (2006).

${ }^{11}$ M. Albrecht, B. Song, and A. Schnurpfeil, J. Appl. Phys. 100, 013702 (2006).

${ }^{12}$ P. Pals and A. Mackinnon, J. Phys.: Condens. Matter 8, 5401 (1996).

${ }^{13}$ M. Galperin and A. Nitzan, Ann. N.Y. Acad. Sci. 1006, 48 (2003).

${ }^{14}$ M. Galperin, A. Nitzan, and M. A. Ratner, Phys. Rev. Lett. 96, 166803 (2006).

${ }^{15}$ A. A. Yanik, G. Klimeck, and S. Datta e-print arXiv:cond-mat/ 0605037.

${ }^{16}$ D. A. Ryndyk and J. Keller, Phys. Rev. B 71, 073305 (2005).

${ }^{17}$ D. A. Ryndyk, M. Hartung, and G. Cuniberti, Phys. Rev. B 73, 045420 (2006).

${ }^{18}$ M. H. Hettler, W. Wenzel, M. R. Wegewijs, and H. Schoeller, Phys. Rev. Lett. 90, 076805 (2003).

${ }^{19}$ E. Bonet, M. M. Deshmukh, and D. C. Ralph, Phys. Rev. B 65, 045317 (2002).

${ }^{20}$ B. Muralidharan, A. W. Ghosh, and S. Datta, Phys. Rev. B 73, 155410 (2006).

${ }^{21}$ W. G. van der Wiel, S. D. Franceschi, J. M. Elzerman, T. Fujisawa, S. Tarucha, and L. P. Kouwenhoven, Rev. Mod. Phys. 75, 1 (2003).

${ }^{22}$ J. N. Pedersen, B. Lassen, A. Wacker, and M. H. Hettler (unpublished).

${ }^{23}$ A. Aviram and M. A. Ratner, Chem. Phys. Lett. 29, 277 (1974).

${ }^{24}$ M. Elbing, R. Ochs, M. Koentopp, M. Fischer, C. von Hanisch, F.
Weigend, F. Evers, H. B. Weber, and M. Mayor, Proc. Natl. Acad. Sci. U.S.A. 102, 8815 (2005).

${ }^{25}$ C. Lacroix, J. Phys. F: Met. Phys. 11, 2389 (1981).

${ }^{26}$ Y. Meir, N. S. Wingreen, and P. A. Lee, Phys. Rev. Lett. 66, 3048 (1991).

${ }^{27}$ Y. Meir, N. S. Wingreen, and P. A. Lee, Phys. Rev. Lett. 70, 2601 (1993).

${ }^{28}$ C. Niu, L.-J. Liu, and T.-H. Lin, Phys. Rev. B 51, 5130 (1995).

${ }^{29}$ S. Lamba and S. K. Joshi, Phys. Rev. B 62, 1580 (2000).

${ }^{30}$ J. J. Palacios, L. Liu, and D. Yoshioka, Phys. Rev. B 55, 15735 (1997).

${ }^{31}$ L. Yi and J.-S. Wang, Phys. Rev. B 66, 085105 (2002).

${ }^{32}$ C. Niu, D. L. Lin, and T.-H. Lin, J. Phys.: Condens. Matter 11, 1511 (1999).

${ }^{33}$ R. Swirkowicz, J. Barnas, and M. Wilczynski, Phys. Rev. B 68, 195318 (2003).

${ }^{34}$ B. R. Bułka and T. Kostyrko, Phys. Rev. B 70, 205333 (2004).

${ }^{35}$ H. Haug and A.-P. Jauho, Quantum Kinetics in Transport and Optics of Semiconductors (Springer, Berlin, 1996).

${ }^{36}$ G. D. Mahan, Many-Particle Physics, 2nd ed. (Plenum, New York, 1990).

${ }^{37}$ L. Kadanoff and G. Baym, Quantum Statistical Mechanics (Benjamin, New York, 1962).

${ }^{38}$ L. V. Keldysh, Sov. Phys. JETP 20, 1018 (1965).

${ }^{39}$ H. S. J. Rammer, Rev. Mod. Phys. 58, 323 (1986).

${ }^{40}$ A. Pecchia and A. Di Carlo, Rep. Prog. Phys. 67, 1497 (2004).

${ }^{41}$ C. W. J. Beenakker, Phys. Rev. B 44, 1646 (1991).

${ }^{42}$ D. V. Averin, A. N. Korotkov, and K. K. Likharev, Phys. Rev. B 44, 6199 (1991).

${ }^{43}$ Y. Meir and N. S. Wingreen, Phys. Rev. Lett. 68, 2512 (1992).

${ }^{44}$ F. Pump and G. Cuniberti, e-print arXiv:cond-mat/0611436, Surf. Sci. (to be published). 